\title{
Giant cross-Kerr nonlinearity in a four-level Y-type atomic system
}

\author{
Nguyen Tuan Anh, ${ }^{1}$ Nguyen Huy Bang, ${ }^{2}$ and Le Van Doai ${ }^{* 2}$ \\ ${ }^{1}$ Ho Chi Minh City University of Food Industry, Vietnam \\ ${ }^{2}$ Vinh University, 182 Le Duan Street, Vinh City, Vietnam.
}

Received January 19, 2021; accepted September 22, 2021; published September 30, 2021

\begin{abstract}
We found the analytical expression for the cross-Kerr nonlinear coefficient in a four-level Y-type atomic system. The analytical model is applied to ${ }^{85} \mathrm{Rb}$ atoms and shows that the cross-Kerr nonlinear coefficient is enhanced by several orders of magnitude under electromagnetically induced transparency. At the same time, the amplitude and the sign of the cross-Kerr nonlinear coefficient are controlled with respect to the intensity and frequency of the coupling laser field. The analytical model can be helpful to explain the experimental results and to study effects in nonlinear optics.
\end{abstract}

The cross-Kerr nonlinear effect, i.e., cross-phase modulation (XPM) is the phenomenon in which the phase of an optical field is modulated by another field. It has beneficial applications in quantum phase gate [1], quantum entanglement state [2], all-optical switching [3], and so on. In such applications, significant nonlinearity is required to achieve nonlinear optical processes with low light intensity.

Today, using electromagnetically induced transparency (EIT) [4], one can get large Kerr nonlinearity in the vicinity of atomic resonance with minimal absorption. Indeed, Schmidt et al. [5] showed giant cross-Kerr nonlinearity in a four-level N-type atomic system under the EIT condition. And then, Kang et al. [6] experimentally verified this model for the $\mathrm{Rb}$ atomic medium. Since then, several schemes have been extensively studied theoretically and experimentally to obtain giant nonlinearity in the four-level atomic systems consisting of inverted Y-type [7], tripod-type [8], M-type [9], and double lambda-type [10] configurations.

Recently, we have developed an analytical model for multi-window EIT in a five-level cascade system [11-13] to study the enhancement and control of Kerr nonlinearity $[14,15]$. The model has also been used to study applications such as optical bistability [16], controlling group velocity $[17,18]$.

In this paper, we propose the analytical model of the four-level Y-type atom to study the enhancement and control of the cross-Kerr nonlinear coefficient. The influence of intensity and frequency of coupling field on the cross-Kerr nonlinear coefficient is investigated.

Figure 1 shows the energy-level diagram for the fourlevel Y-type atomic system. The $|1\rangle \leftrightarrow|2\rangle$ transition is applied by the probe laser beam with frequency $\omega_{p}$, the

\footnotetext{
*E-mail: doailv@vinhuni.edu.vn
}

$|2\rangle \leftrightarrow|3\rangle$ transition is coupled by the coupling laser beam with frequency $\omega_{c}$ and the $|2\rangle \leftrightarrow|4\rangle$ transition is driven by the signal laser beam with frequency $\omega_{s}$. In this configuration, the coupling beam can generate the EIT effect for the probe beam, while the signal beam can induce the cross-Kerr effect for the probe beam.

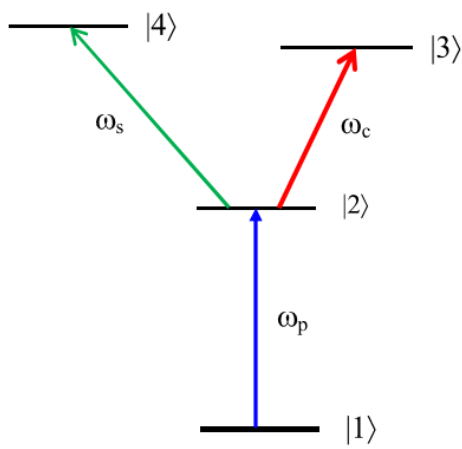

Fig. 1. Energy-level diagram for the four-level Y-type atomic system.

The density matrix equations of motion for the fourlevel Y-type atoms in the laser beams are given by:

$$
\begin{aligned}
\&_{44}= & -\left(\Gamma_{43}+\Gamma_{42}\right) \rho_{44}+\frac{i}{2} \Omega_{s}\left(\rho_{42}-\rho_{24}\right), \\
\&_{33}= & \Gamma_{43} \rho_{44}-\Gamma_{32} \rho_{33}+\frac{i}{2} \Omega_{c}\left(\rho_{32}-\rho_{23}\right), \\
\&_{22}= & \Gamma_{42} \rho_{44}+\Gamma_{32} \rho_{33}-\Gamma_{21} \rho_{22}+\frac{i}{2} \Omega_{p}\left(\rho_{21}-\rho_{12}\right) \\
& +\frac{i}{2} \Omega_{c}\left(\rho_{23}-\rho_{32}\right)+\frac{i}{2} \Omega_{s}\left(\rho_{24}-\rho_{42}\right) \\
\&_{11}= & \Gamma_{21} \rho_{22}+\frac{i}{2} \Omega_{p}\left(\rho_{12}-\rho_{21}\right), \\
\&_{43}= & -\left[i\left(\Delta_{s}-\Delta_{c}\right)+\gamma_{43}\right] \rho_{43}-\frac{i \Omega_{s}}{2} \rho_{23}+\frac{i \Omega_{c}}{2} \rho_{42}, \\
\&_{42}= & {\left[i \Delta_{s}-\gamma_{42}\right] \rho_{42}+\frac{i \Omega_{s}}{2}\left(\rho_{44}-\rho_{22}\right)+\frac{i \Omega_{c}}{2} \rho_{43}+\frac{i \Omega_{p}}{2} \rho_{41}, } \\
\&_{41}= & -\left[i\left(\Delta_{p}+\Delta_{s}\right)+\gamma_{41}\right] \rho_{41}-\frac{i \Omega_{s}}{2} \rho_{21}+\frac{i \Omega_{p}}{2} \rho_{42}, \\
\&_{32}= & -\left(\gamma_{32}-i \Delta_{c}\right) \rho_{32}+\frac{i \Omega_{c}}{2}\left(\rho_{33}-\rho_{22}\right)+\frac{i \Omega_{p}}{2} \rho_{31}, \\
\&_{31}= & -\left[\gamma_{31}-i\left(\Delta_{p}+\Delta_{c}\right)\right] \rho_{31}+\frac{i \Omega_{p}}{2} \rho_{32}-\frac{i \Omega_{c}}{2} \rho_{21},
\end{aligned}
$$


$\&_{21}=-\left(\gamma_{21}-i \Delta_{p}\right) \rho_{21}+\frac{i \Omega_{p}}{2}\left(\rho_{22}-\rho_{11}\right)-\frac{i \Omega_{c}}{2} \rho_{31}-\frac{i \Omega_{s}}{2} \rho_{41},(10)$

$\rho_{k i}=\rho_{i k}^{*}$,

$\rho_{11}+\rho_{22}+\rho_{33}+\rho_{44}=1$,

where $\Omega_{\mathrm{p}}=d_{31} E_{p} / \hbar, \Omega_{\mathrm{c}}=d_{32} E_{c} / \hbar$ and $\Omega_{\mathrm{s}}=d_{42} E_{s} / \hbar$ are Rabi frequencies of the probe, coupling and signal beam, with $d_{k i}$ is the dipole matrix moments induced on the $|k\rangle-|i\rangle$ transition; $\Delta_{\mathrm{p}}=\omega_{\mathrm{p}}-\omega_{31}, \Delta_{\mathrm{c}}=\omega_{\mathrm{c}}-\omega_{32}$ and $\Delta_{\mathrm{s}}=\omega_{\mathrm{s}}-\omega_{42}$ are the frequency detunings of the probe, coupling and signal beams; $\Gamma_{k i}$ is the population decay rate from the level $|k\rangle$ to the level $|i\rangle$, while $\gamma_{k i}=\Gamma_{k i} / 2$ is the coherence decay rate.

Under the weak probe field approximation, we have found the solution for the density matrix element $\rho_{21}$ as follows:

$$
\rho_{21}=-\frac{i \Omega_{p} / 2}{\gamma_{21}-i \Delta_{p}+\frac{\Omega_{c}^{2} / 4}{\gamma_{31}-i\left(\Delta_{p}+\Delta_{c}\right)}+\frac{\Omega_{s}^{2} / 4}{\gamma_{41}-i\left(\Delta_{p}+\Delta_{s}\right)}} .
$$

The total susceptibility for the probe field can be written as [11]:

$$
\begin{aligned}
\chi_{p} & =-2 \frac{N d_{21}}{\varepsilon_{0} E_{p}} \rho_{21} \\
& \equiv \frac{N d_{21}}{\varepsilon_{0} E_{p}} \frac{i \Omega_{p}}{\gamma_{21}-i \Delta_{p}+\frac{\Omega_{c}^{2} / 4}{\gamma_{31}-i\left(\Delta_{p}+\Delta_{c}\right)}+\frac{\Omega_{s}^{2} / 4}{\gamma_{41}-i\left(\Delta_{p}+\Delta_{s}\right)}}
\end{aligned}
$$

with $N$ is the atomic density, $\varepsilon_{0}$ is the vacuum permittivity.

We now make the Taylor expansion of the expression (14) over $\Omega_{\mathrm{s}}$ to extract the linear and nonlinear terms of the susceptibility. We have:

$$
\chi=\frac{i N d_{21}^{2}}{\varepsilon_{0} \mathrm{~h}} \frac{1}{A}-\left[\frac{i N d_{21}^{2} d_{42}^{2}}{\varepsilon_{0} \mathrm{~h}^{3}} \frac{1}{A^{2} B}\right] E_{s}^{2} .
$$

where:

$$
\begin{aligned}
& A=\gamma_{21}-i \Delta_{p}+\frac{\Omega_{c}^{2} / 4}{\gamma_{31}-i\left(\Delta_{p}+\Delta_{c}\right)}, \\
& B=\gamma_{41}-i\left(\Delta_{p}+\Delta_{s}\right) .
\end{aligned}
$$

On the other hand, the total susceptibility (15) can be written in another form as [11]:

$$
\chi=\chi^{(1)}+3 E_{s}^{2} \chi^{(3)} .
$$

with $E_{s}$ being the electric field of a signal beam.

By comparing Eqs. (15) and (18), we obtain the expressions of first- and third- order susceptibilities for the probe beam as:

$$
\begin{gathered}
\chi_{p}^{(1)}=\frac{i N d_{21}^{2}}{\mathrm{~h} \varepsilon_{0}} \frac{1}{A}, \\
\chi_{p}^{(3)}=\frac{i N d_{21}^{2} d_{42}^{2}}{6 \mathrm{~h}^{3} \varepsilon_{0}} \frac{1}{A^{2} B} .
\end{gathered}
$$

http://www.photonics.pl/PLP
The linear dispersion $n_{0}$ and cross-Kerr nonlinear coefficient $n_{2}$ of the four-level Y-type atomic medium for the probe beam are determined by:

$$
\begin{aligned}
& n_{0}=1+\frac{\operatorname{Re}\left(\chi_{p}^{(1)}\right)}{2}, \\
& n_{2}=\frac{3 \operatorname{Re}\left(\chi_{p}^{(3)}\right)}{2 \varepsilon_{0} n_{0}^{2} c} .
\end{aligned}
$$

Here, we use the ${ }^{85} \mathrm{Rb}$ atoms with the $|1\rangle,|2\rangle,|3\rangle$ and $|4\rangle$ states as $5 \mathrm{~S}_{1 / 2}(F=2), 5 \mathrm{P}_{3 / 2}\left(F^{\prime}=3\right), 5 \mathrm{D}_{5 / 2}\left(F^{\prime \prime}=2\right)$ and $5 \mathrm{D}_{5 / 2}\left(F^{\prime \prime}=3\right)$, respectively [19]. The employed parameters as [15]: $N=10^{12}$ atoms $/ \mathrm{cm}^{3} ; \Gamma_{32}=\Gamma_{42}=0.97$ $\mathrm{MHz} ; \Gamma_{21}=6 \mathrm{MHz} ; d_{21}=1.62 \times 10^{-29}$ C.m; $\omega_{\mathrm{p}}=3.84 \times 10^{8}$ $\mathrm{MHz}$.

First of all, in order to see the enhancement of crossKerr nonlinearity $n_{2}$ under the EIT condition we plot $n_{2}$ versus the probe detuning in two cases of the absence $\left(\Omega_{\mathrm{c}}\right.$ $=0)$ and presence $\left(\Omega_{\mathrm{c}}=3 \mathrm{MHz}\right)$ of the coupling beam as in Fig. 2. The dash-dotted line in Fig. 2 represents the EIT spectrum when Rabi frequency $\Omega_{\mathrm{c}}=3 \mathrm{MHz}$. We can see from Figure 2 that the nonlinear coefficient is significantly increased on either side of the EIT window at $\Delta_{p}=0$. That is to say, a pair of positive-negative peaks of $n_{2}$ appears around resonance frequency of the probe field. The amplitude of the nonlinear coefficient in the presence of EIT is increased by several orders compared to that in the absence of EIT.

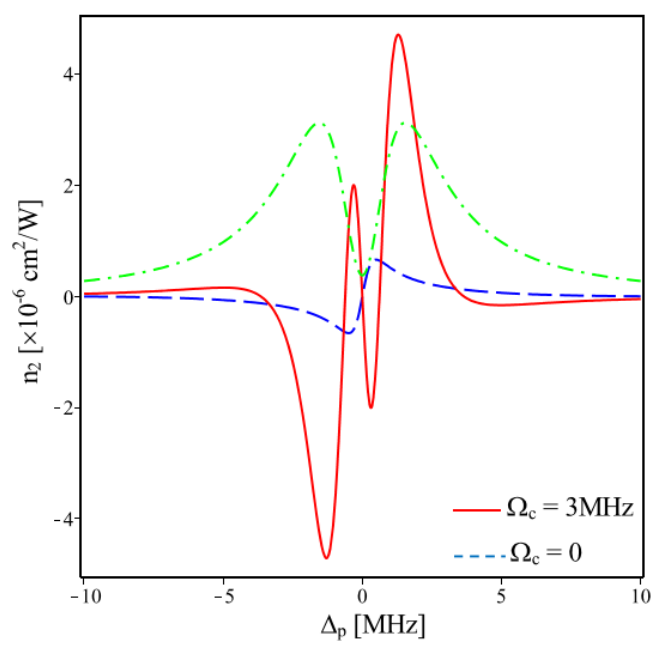

Fig. 2. Variations of the cross-Kerr nonlinear coefficient versus probe detuning $\Delta_{\mathrm{p}}$ when $\Omega_{\mathrm{c}}=0$ (dashed line) and $\Omega_{\mathrm{c}}=3 \mathrm{MHz}$ (solid line), and $\Delta_{\mathrm{c}}=\Delta_{\mathrm{s}}=0$. The dash-dotted line represents the EIT spectrum when Rabi frequency $\Omega_{\mathrm{c}}=3 \mathrm{MHz}$.

Next, in Fig. 3 we consider the control of the nonlinear coefficient via the coupling intensity by plotting $n_{2}$ versus the coupling Rabi frequency at probe frequency $\Delta_{\mathrm{p}}= \pm 2$ $\mathrm{MHz}$, which corresponds to the maximum and minimum points around the EIT window. It is clear that the magnitude and the sign of the nonlinear coefficient are changed with respective to coupling laser intensity. 


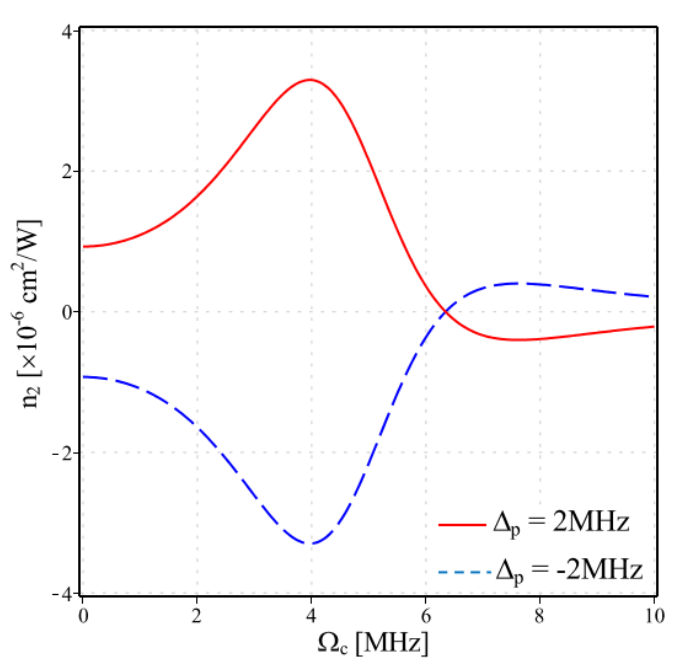

Fig. 3. Variations of the cross-Kerr nonlinear coefficient versus the coupling Rabi frequency $\Omega_{\mathrm{c}}$ when $\Delta_{\mathrm{p}}=-2 \mathrm{MHz}$ (dashed line) and $\Delta_{\mathrm{p}}=2$ $\mathrm{MHz}$ (solid line), and $\Delta_{\mathrm{c}}=\Delta_{\mathrm{s}}=0$.

Finally, in Fig. 4 we consider the variation of the nonlinear coefficient via coupling frequency detuning $\Delta_{\mathrm{c}}$ when $\Delta_{\mathrm{p}}=\Delta_{\mathrm{s}}=0$ and $\Omega_{\mathrm{c}}=2 \mathrm{MHz}$. The variation in Fig. 4 shows that the cross-Kerr nonlinear coefficient of medium also exhibits a pair of positive-negative peaks around $\Delta_{\mathrm{c}}=$ 0 . The magnitude and sign of the nonlinear coefficient are also controlled concerning the coupling laser frequency.

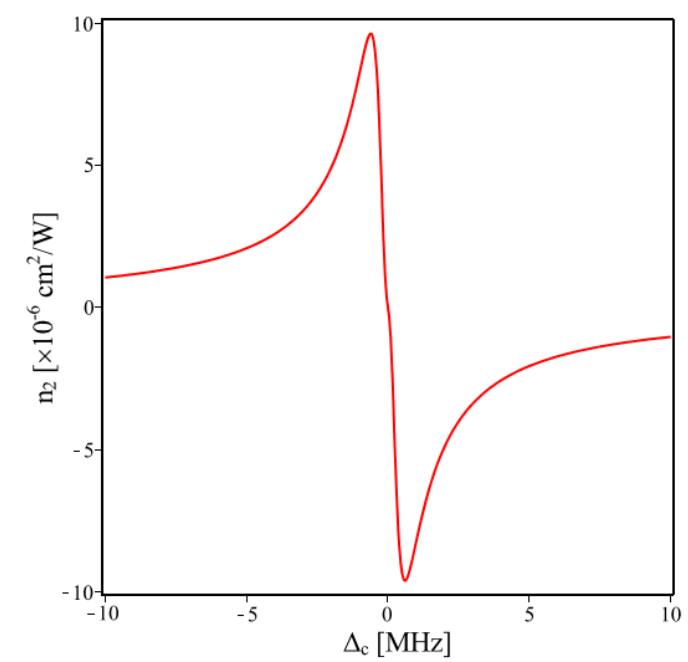

Fig. 4. Variation of the cross-Kerr nonlinear coefficient via coupling detuning $\Delta_{\mathrm{c}}$ when $\Omega_{\mathrm{c}}=3 \mathrm{MHz}$ and $\Delta_{\mathrm{p}}=\Delta_{\mathrm{s}}=0$.

In conclusions, the enhancement and control of crossKerr nonlinearity in a four-level Y-type atomic system have been studied by analytic methods. It is shown that the cross-Kerr nonlinear coefficient of a four-level Y-type atomic system is significantly enhanced around the transparency window. Significantly, the magnitude and sign of the nonlinear coefficient are controlled by tuning the intensity and frequency of the coupling laser. Such an analytical model has an important role to play for experimental observation or implementation of related studies such as slow light, polarization phase gate, alloptical switching and so on.

This work has been sponsored and funded by Ho Chi Minh City University of Food Industry under Contract No.148/ HD-DCT.

\section{References}

[1] C. Ottaviani, D. Vitali, M. Artoni, F. Cataliotti, P. Tombesi, Phys. Rev. Lett. 90, 197902 (2003).

[2] C. Zhu, G. Huang, Opt. Express 19, 23364 (2011).

[3] C. Hang, G. Huang, Opt. Express 18, 2952 (2010).

[4] M. Fleischhauer, I. Mamoglu, J.P. Marangos, Rev. Mod. Phys. 77, 633 (2005).

[5] H. Schmidt, A. Imamogdlu, Opt. Lett. 21, 1936 (1996).

[6] H. Kang And Y. Zhu, Phys. Rev. Lett. 91, 093601 (2003).

[7] J. Kou, R.G. Wan, Z.H. Kang, H.H. Wang, L. Jiang, X.J. Zhang, Y. Jiang, J.Y. Gao, J. Opt. Soc. Am. B. 27, 2035 (2010).

[8] X. Yang, S. Li, C. Zhang, H. Wang, J. Opt. Soc. Am. B. 26, 1423 (2009).

[9] C. Ottaviani, D. Vitali, M. Artoni, F. Cataliotti, P. Tombesi, Phys. Rev. Lett. 90, 197902 (2003).

[10] H. Sun, Y. Niu, S. Jin, S. Gong, J. Phys. B: At. Mol. Opt. Phys. 40, 3037 (2007).

[11] L.V. Doai, P.V. Trong, D.X. Khoa, N.H. Bang, Optik, 125, 3666 (2014).

[12] D.X. Khoa, P.V. Trong, L.V. Doai, N.H. Bang, Phys, Scr. 91, 035401 (2016).

[13] D.X. Khoa, L.C. Trung, P.V. Thuan, L.V. Doai, N.H. Bang, J. Opt. Soc. Am. B. 34(6), 1255 (2017).

[14] D.X. Khoa, L.V. Doai, D.H. Son, N.H. Bang, J. Opt. Soc. Am. B. 31, 1330 (2014).

[15] L.V. Doai, N.L.T. An, D.X. Khoa, V.N. Sau, N.H. Bang, J. Opt. Soc. Am. B. 36, 2856 (2019).

[16]D.X. Khoa, L.V. Doai, L.N.M. Anh, L.C. Trung, P.V. Thuan, N.T. Dung, N.H. Bang, J. Opt. Soc. Am. B. 33, 735 (2016).

[17] N.T. Anh, L.V. Doai, N.H. Bang, J. Opt. Soc. Am. B. 35, 1233 (2018).

[18] N.T. Anh, L.V. Doai, D.H. Son, N.H. Bang, Optik 171, 721(2018).

[19] D.A. Steck, $\mathrm{Rb}^{85}$ D Line Data: http://Steck.Us/Alkalidata/rubidium85numbers.pdf 